\title{
Difusividade efetiva de grãos e frutos de pinhão-manso
}

\section{Effective diffusivity of jatropha grains and fruits}

\author{
Valdiney Cambuy Siqueira ${ }^{1 *}$; Osvaldo Resende ${ }^{2}$; Tarcísio Honório Chaves ${ }^{3}$
}

\section{Resumo}

Objetivou-se com o presente trabalho verificar a influência da contração volumétrica na determinação do coeficiente de difusão efetivo, bem como obter a energia de ativação dos grãos e dos frutos de pinhão-manso submetidos à secagem em diversas condições de ar. Foram utilizados grãos e frutos de pinhão-manso, com teor de água de 0,50 e 4,40 decimal base seca (b.s), respectivamente, e submetidos à secagem nas temperaturas de $45,60,75,90$ e $105^{\circ} \mathrm{C}$ e umidade relativa do ar de secagem de 14,$5 ; 7,4$; 3,$8 ; 2,2$ e 1,4\% para os frutos e 15,6; 7,4; 4,0;1,8 e 1,2\%, para os grãos. O volume dos grãos e dos frutos de pinhão-manso, considerados esferoides, foi obtido por meio da medição dos três eixos ortogonais ao longo da redução do teor de água e a contração volumétrica avaliada por meio da redução do raio equivalente com o processo de secagem. Conclui-se que, o coeficiente de difusão efetivo dos grãos e dos frutos de pinhão-manso aumenta com a elevação da temperatura do ar, a inclusão da contração volumétrica promove um melhor ajuste do modelo da difusão líquida e a energia de ativação dos grãos e dos frutos de pinhão-manso são similares sendo que a inclusão da contração volumétrica praticamente não altera este parâmetro.

Palavras-chave: Temperaturas, contração volumétrica, coeficiente de difusão efetivo

\begin{abstract}
This study aimed to assess the influence of volumetric shrinkage in determining the effective diffusion coefficient, and obtain the activation energy of jatropha grains and fruits subjected to drying under varying air conditions. Jatropha grains and fruits were used with moisture content of 0.50 and decimal dry basis $4.40(\mathrm{db})$, respectively, dried temperatures of $45,60,75,90$, and $105^{\circ} \mathrm{C}$, and drying air relative humidity of $14.5,7.4,3.8,2.2$, and $1.4 \%$ for fruits and $15.6,7.4,4.0,1.8$, and $1.2 \%$ for grains. The volume of jatropha grains and fruits, considered spheroids, was obtained by measuring the three orthogonal axes during moisture content reduction, and volumetric shrinkage was assessed by reducing the equivalent radius with the drying process. It is concluded that the effective diffusion coefficient of jatropha grains and fruits increases with increasing air temperature; the inclusion of volumetric shrinkage promotes a better fit of the liquid diffusion model; and activation energy of Jatropha grains and fruits is similar but the inclusion of volumetric shrinkage practically did not change this parameter.
\end{abstract}

Key words: Temperatures, volumetric shrinkage, effective diffusion coefficient

${ }^{1}$ Doutorando em Engenharia Agrícola, Universidade Federal de Lavras, Lavras, MG. E-mail: vcambuy@yahoo.com

${ }^{2}$ Eng ${ }^{\mathrm{O}}$ Agrícola, Prof. Dr. do Instituto Federal de Educação, Ciência e Tecnologia Goiano - Campus Rio Verde, E-mail: osvresende@ yahoo.com.br

${ }^{3}$ Discente em Agronomia, Instituto Federal de Educação, Ciência e Tecnologia Goiano - Campus Rio Verde. Bolsista PIBIC/ CNPq. E-mail: tarcisio.chaves@hotmail.com

*Autor para correspondência 


\section{Introdução}

Dentre as etapas pós-colheita a secagem assume uma enorme importância, pois está relacionada diretamente com a qualidade final do produto, uma vez que visa a redução do teor de água até níveis seguros, que possibilita a antecipação da colheita, disponibilizando a área para novos cultivos, dificultando e/ou inibindo o crescimento de microrganismos, reduz a proliferação dos insetos e a atividade metabólica da semente, mantendo-a viável por um maior período e facilitando o armazenamento.

A secagem é um complexo processo que envolve uma transferência simultânea de calor e massa e que pode resultar em mudanças significativas na composição química, estrutura e propriedades físicas dos alimentos (KOÇ; EREN; ERTEKIN, 2008). Segundo Corrêa et al. (2006a), esse fenômeno envolve o movimento da água do interior para a periferia do grão por meio de mecanismos distintos.

A representação matemática do processo de secagem de diversos produtos agrícolas vem, há algum tempo, sendo estudada e utilizada na tentativa de predizer os fenômenos que acontecem durante este processo (CORRÊA et al., 2006b). Informações referentes ao assunto são de extrema importância para auxiliar no desenvolvimento de equipamentos e adaptações daqueles já existentes, visando a obter maior rendimento nesta etapa fundamental durante o período pós-colheita.

De acordo com Goneli et al. (2007), os modelos de secagem baseados na teoria da difusão líquida têm merecido atenção especial por parte dos pesquisadores. Segundo Goneli et al. (2009), a difusão de água em produtos agrícolas durante a secagem é um processo complexo que pode envolver diferentes mecanismos, como a difusão molecular, difusão capilar, difusão de superfície, fluxo hidrodinâmico, difusão de vapor e difusão térmica. A teoria da difusão líquida assume que não há influência da capilaridade, despreza os efeitos da transferência de energia e massa de um corpo para outro, e também considera que os corpos entram em equilíbrio térmico com o ar instantaneamente.

Pode-se dizer que o coeficiente de difusão efetivo descreve a velocidade de saída de água do grão. Como a difusividade varia conforme mudam as condições de secagem, ela não é intrínseca ao material, assim, convenciona-se chamá-la de difusividade efetiva (OLIVEIRA; OLIVEIRA; PARK, 2006).

Alguns pesquisadores, ao analisarem o modelo da difusão líquida para a representação do processo de secagem, consideraram em seus trabalhos o efeito da contração volumétrica no melhor ajuste do modelo, (RAMOS; BRANDÃO; SILVA, 2005; RESENDE et al., 2007; GONELI et al., 2007).

Considerando a importância do estudo teórico do processo de secagem dos produtos agrícolas, o destaque da cultura do pinhão-manso como oleaginosa e da falta de informações a respeito do comportamento das sementes e dos frutos desta cultura durante a redução de água, o presente trabalho foi realizado com o objetivo determinar e avaliar o coeficiente de difusão efetivo acrescentando o efeito da contração volumétrica, bem como obter a energia de ativação para o processo de secagem dos grãos e dos frutos de pinhão-manso, durante à secagem em diversas condições de ar.

\section{Material e Métodos}

O experimento foi desenvolvido no Laboratório de Pós-colheita de Produtos Vegetais do Instituto Federal de Educação, Ciência e Tecnologia Goiano Campus Rio Verde (IF Goiano - Campus Rio Verde). Utilizaram-se grãos e frutos de pinhão-manso, com teor de água de 0,50 e 4,40 decimal base seca (b.s.), respectivamente. Os materiais foram submetidos à secagem em estufa com ventilação de ar forçada nas cinco condições de temperatura (T) de 45, 60, 75, 90 e $105{ }^{\circ} \mathrm{C}$ e umidades relativas de 14,$5 ; 7,4 ; 3,8 ; 2,2$ e $1,4 \%$ para os frutos e 15,$6 ; 7,4 ; 4,0 ; 1,8$ e $1,2 \%$, para os grãos. A temperatura e a umidade relativa do 
ar de secagem foram monitoradas por meio de um psicrômetro instalado no interior da estufa. O sensor do psicrômetro opera na faixa de temperatura entre -50 e $200{ }^{\circ} \mathrm{C}$, com resolução de $0,1^{\circ} \mathrm{C}$.

$\mathrm{O}$ processo de secagem foi interrompido quando os grãos e frutos atingiram o teor de água de 0,10 $\pm 0,006$ (b.s), determinado em estufa a $105 \pm 1{ }^{\circ} \mathrm{C}$, durante 24 horas, em três repetições (BRASIL, 2009).

Para a determinação da razão do teor de água dos grãos e frutos de pinhão-manso, para as diferentes condições de secagem, utilizou-se a seguinte expressão:

$$
R X=\frac{X^{*}-X_{e}^{*}}{X_{i}^{*}-X_{e}^{*}}
$$

em que,

$\mathrm{RX}$ : razão de teor de água do produto, adimensional;

$\mathrm{X}^{*}$ : teor de água do produto (b.s);

$\mathrm{X}_{\mathrm{i}}^{*}$ : teor de água inicial do produto (b.s);

$\mathrm{X}_{\mathrm{e}}^{*}$ : teor de água de equilíbrio do produto (b.s).

A redução do teor de água, ao longo da secagem, foi acompanhada pelo método gravimétrico (perda de massa), conhecendo-se o teor de água inicial do produto, até atingir o teor de água desejado. $\mathrm{O}$ acompanhamento da redução da massa durante a secagem foi realizado com auxílio de uma balança analítica com resolução de $0,01 \mathrm{~g}$.

O modelo da difusão líquida para a forma geométrica cilíndrica, com aproximação de oito termos (Equação 2), foi ajustado aos dados experimentais de secagem dos grãos e frutos de pinhão-manso considerando $\mathrm{o}$ raio equivalente inicial dos mesmos e mantendo-o constante ao longo da secagem e, o raio equivalente considerando-se a contração volumétrica dos materiais.

$$
\mathrm{RX}=\frac{\mathrm{X}^{*}-\mathrm{X}_{\mathrm{e}}^{*}}{\mathrm{X}_{\mathrm{i}}^{*}-\mathrm{X}_{\mathrm{e}}^{*}}=\sum_{\mathrm{n}=1}^{\infty} \frac{4}{\lambda_{\mathrm{n}}^{2}} \exp \left[-\frac{\lambda_{\mathrm{n}}^{2} \cdot \mathrm{D} \cdot \mathrm{t}}{\mathrm{r}}\right]
$$

em que,

D: coeficiente de difusão efetivo, $\mathrm{m}^{2} \cdot \mathrm{s}^{-1}$;

n: número de termos;

$\lambda_{\mathrm{n}}$ : raízes da equação de Bessel de ordem zero;

r: raio de esfera equivalente, $\mathrm{m}$;

t: tempo de secagem, $h$.

Para o monitoramento da contração volumétrica, utilizaram-se 15 grãos e 15 frutos de pinhão-manso, individualizados em cápsulas de alumínio com dimensões de: 60,12 mm de diâmetro e 41,0 mm de altura. A cada intervalo pré-definido de teor de água os grãos e os frutos eram medidos nas três posições (comprimento, largura e espessura) com o auxílio de um paquímetro digital com resolução de 0,01 $\mathrm{mm}$.

O volume dos grãos e dos frutos de pinhãomanso foi obtido ao longo do processo de secagem de acordo com a expressão proposta por Mohsenin (1986):

$$
\mathrm{V}=\frac{\pi \cdot \mathrm{a} \cdot \mathrm{b} \cdot \mathrm{c}}{6}
$$

em que,

$\mathrm{V}$ : volume dos grãos e dos frutos, $\mathrm{mm}^{3}$;

a: comprimento, $\mathrm{mm}$;

b: largura, mm; e

c: espessura, mm.

As dimensões características dos eixos ortogonais dos grãos e dos frutos de pinhão-manso foram determinadas com os mesmos na sua posição natural de repouso, como ilustrado na Figura 1. 
Figura 1. Desenho esquemático dos grãos e dos frutos de pinhão-manso, considerados esferoides, com suas dimensões características.

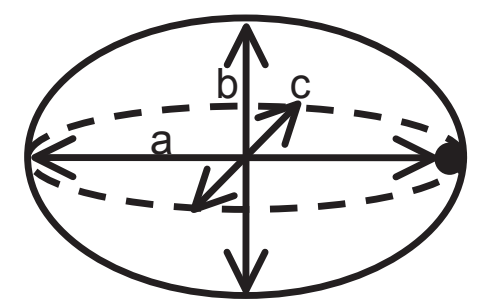

Fonte: Elaboração dos autores.

$\mathrm{O}$ raio da esfera equivalente foi calculado pela seguinte expressão:

$$
r=\sqrt[3]{\frac{3 \cdot V}{4 \cdot \pi}}
$$

A relação do aumento do coeficiente de difusão efetivo, com a elevação da temperatura do ar de secagem foi descrita por meio da equação de Arrhenius.

$$
\mathrm{D}=\mathrm{D}_{\mathrm{o}} \cdot \exp \left(\frac{-\mathrm{E}_{\mathrm{a}}}{\mathrm{R} \cdot \mathrm{T}_{\mathrm{a}}}\right)
$$

em que,

Do: fator pré-exponencial;

Ea: energia de ativação, $\mathrm{kJ} \cdot \mathrm{mol}^{-1}$;

$\mathrm{R}$ : constante universal dos gases, 8,134 kJ.kmol${ }^{1} \cdot \mathrm{K}^{-1}$

Ta: temperatura absoluta, $\mathrm{K}$.

Os coeficientes da expressão de Arrhenius podem ser facilmente obtidos linearizando a Equação 5 com a aplicação do logaritmo na seguinte forma:

$$
\mathrm{LnD}=\operatorname{LnD}_{\mathrm{o}}-\frac{\mathrm{E}_{\mathrm{a}}}{\mathrm{R}} \cdot \frac{1}{\mathrm{~T}_{\mathrm{a}}}
$$

Após a identificação do modelo para a representação da difusividade efetiva dos grãos e dos frutos de pinhão-manso, promoveu-se o teste da hipótese de igualdade dos modelos utilizando a técnica de identidade de modelos descrita por Regazzi (2003), que permite analisar a equivalência entre os modelos utilizados, objetivando o ajuste de uma única equação para descrição do fenômeno estudado.

Os parâmetros dos modelos Linear, utilizados para descrever o efeito da contração volumétrica na difusividade efetiva dos grãos e dos frutos de pinhão-manso ( $a_{i}$ e $\left.b_{i}\right)$, foram comparados entre si para verificar a sua igualdade. As seguintes hipóteses foram formuladas:

Modelo Linear:

$\mathrm{H}_{\mathrm{o}}: \mathrm{a}_{1}=\mathrm{a}_{2}$ e $\mathrm{b}_{1}=\mathrm{b}_{2}$.

versus

$\mathrm{H}_{\mathrm{a}}$ : existe pelo menos uma desigualdade entre os parâmetros.

\section{Resultados e Discussão}

Na Figura 2 "A" estão apresentados os valores da razão de teor de água dos grãos de pinhão-manso, submetidos à secagem em diversas condições de ar. Nota-se que o tempo necessário para que os grãos de pinhão-manso atingissem o teor de água de 0,10 $\pm 0,006$ (b.s), foi de 7,$11 ; 3,90 ; 2,60 ; 1,79$ e 1,26 h para as temperaturas de secagem de $45,60,75$, 
90 e $105^{\circ} \mathrm{C}$, respectivamente. Logo, o aumento da temperatura do ar promove redução no tempo de secagem dos grãos. O mesmo comportamento foi observado para a secagem dos frutos de pinhãomanso que, necessitaram de 10,28; 10,98; 15,26; 21,83 e $38,62 \mathrm{~h}$ para as temperaturas de secagem de $105,90,75,60$ e $45^{\circ} \mathrm{C}$, respectivamente, para atingir o teor de água de 4,40 (b.s), (Figura 2 "B”).

Com o aumento da temperatura do ar há uma maior diferença entre a pressão de vapor d'agua do ar de secagem e do produto, fazendo com que a água seja removida com mais facilidade e rapidez, fato observado por diversos pesquisadores para inúmeros produtos (LAHSASNI et al., 2004; MOHAPATRA; RAO, 2005; GELY; SANTALLA, 2007; SIRISOMBOON; KITCHAIYA, 2009; MIRANDA et al., 2009; ULLMANN et al., 2010; VEGA-GÁLVEZ et al., 2011; SOUSA et al., 2011).

Analisando a Figura 2 "A" e "B", verifica-se que a razão de teor de água dos grãos de pinhão-manso após a secagem ficou em torno de 0,16 , enquanto que a dos frutos foi de aproximadamente 0,01 , este comportamento está associado ao alto teor de água que os frutos apresentavam antes da secagem em relação aos grãos.

Figura 2. Valores experimentais da razão de teor de água dos grãos (A) e frutos (B) de pinhão-manso submetidos à secagem em diversas condições de ar.

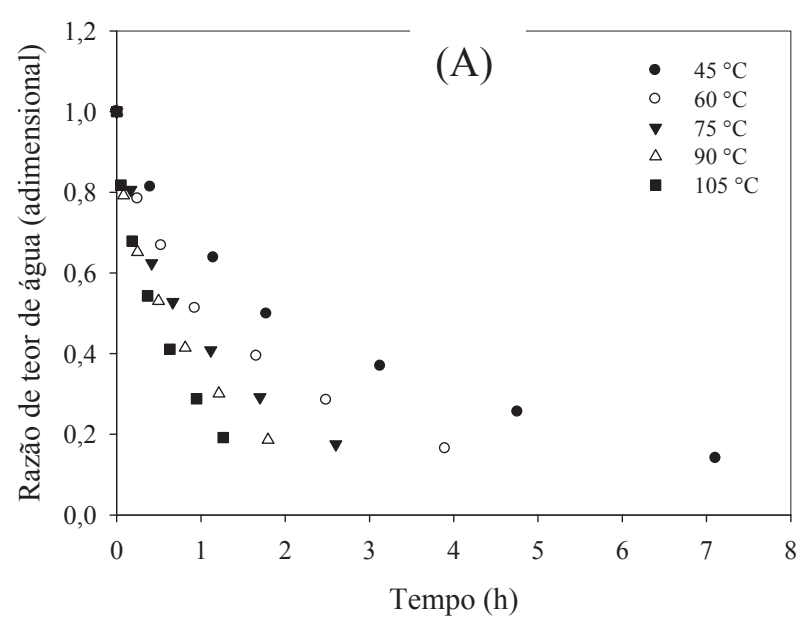

Fonte: Elaboração dos autores.

Os valores do raio equivalente dos grãos e dos frutos de pinhão-manso, utilizados no modelo da difusão líquida para a determinação do coeficiente de difusão efetivo durante a secagem em diversas condições de ar, são apresentados na Tabela 1. Observa-se que, os valores do raio equivalente dos grãos e dos frutos diminuíram com a redução do teor de água, devido, essencialmente, a contração volumétrica dos materiais durante a secagem, concordando com os resultados obtidos por Corrêa et al. (2006a), Goneli et al. (2007), Resende et al. (2007), que pesquisaram com grãos de feijão, trigo e feijão respectivamente.

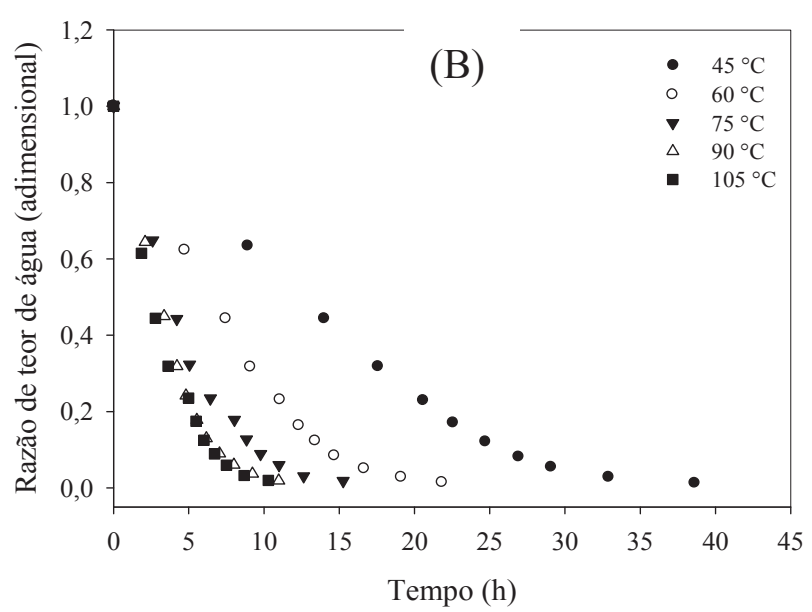

Verifica-se na Tabela 1 , que o raio equivalente dos frutos de pinhão-manso reduziram em média $9,7 \times 10^{-3} \mathrm{~m}$, com o processo de secagem, enquanto que o raio equivalente dos grãos de pinhão-manso apresentaram uma redução média de $0,57 \times 10^{-3} \mathrm{~m}$. Este comportamento é decorrente da diferença entre a forma, o tamanho e o teor de água dos frutos e dos grãos de pinhão-manso utilizados. O que explica também, o fato dos valores do raio equivalente dos frutos de pinhão-manso serem extremamente maiores que os dos grãos. 
Tabela 1. Valores do raio equivalente $\left(\mathrm{r}, \mathrm{x} 10^{-3} \mathrm{~m}\right)$ e do teor de água $\left(\mathrm{X}^{*}, \mathrm{~b} . \mathrm{s}\right)$ dos grãos e dos frutos de pinhão-manso, obtidos durante a secagem nas temperaturas de $45,60,75,90$ e $105^{\circ} \mathrm{C}$.

\begin{tabular}{|c|c|c|c|c|c|c|c|c|c|}
\hline \multicolumn{10}{|c|}{ Frutos } \\
\hline \multicolumn{2}{|c|}{$45^{\circ} \mathrm{C}$} & \multicolumn{2}{|c|}{$60^{\circ} \mathrm{C}$} & \multicolumn{2}{|c|}{$75^{\circ} \mathrm{C}$} & \multicolumn{2}{|c|}{$90^{\circ} \mathrm{C}$} & \multicolumn{2}{|c|}{$105^{\circ} \mathrm{C}$} \\
\hline $\mathrm{X}^{*}$ & $\mathrm{r}$ & $\mathrm{X}^{*}$ & $\mathrm{r}$ & $\mathrm{X}^{*}$ & $\mathrm{r}$ & $\mathrm{X}^{*}$ & $\mathrm{r}$ & $\mathrm{X}^{*}$ & $\mathrm{r}$ \\
\hline 4,40 & 34,31 & 4,40 & 34,09 & 4,40 & 33,77 & 4,40 & 34,29 & 4,40 & 34,22 \\
\hline 2,80 & 30,76 & 2,75 & 30,82 & 2,86 & 30,11 & 2,84 & 30,00 & 2,71 & 28,55 \\
\hline 1,90 & 29,08 & 1,97 & 28,85 & 1,96 & 28,09 & 1,99 & 27,67 & 1,97 & 27,55 \\
\hline 1,43 & 27,74 & 1,41 & 27,53 & 1,44 & 27,06 & 1,42 & 26,61 & 1,42 & 26,18 \\
\hline 1,04 & 26,73 & 1,04 & 26,96 & 1,05 & 26,20 & 1,08 & 26,08 & 1,05 & 25,89 \\
\hline 0,79 & 25,98 & 0,75 & 26,10 & 0,81 & 25,65 & 0,80 & 25,53 & 0,79 & 25,57 \\
\hline 0,57 & 25,38 & 0,57 & 25,50 & 0,58 & 25,06 & 0,59 & 25,27 & 0,57 & 25,38 \\
\hline 0,40 & 24,95 & 0,40 & 25,03 & 0,42 & 24,61 & 0,42 & 25,08 & 0,41 & 25,23 \\
\hline 0,28 & 24,53 & 0,25 & 24,58 & 0,29 & 24,42 & 0,29 & 25,00 & 0,28 & 25,11 \\
\hline 0,17 & 24,29 & 0,15 & 24,37 & 0,16 & 24,30 & 0,18 & 24,90 & 0,17 & 24,92 \\
\hline 0,10 & 23,90 & 0,10 & 24,25 & 0,11 & 24,32 & 0,11 & 24,86 & 0,11 & 24,83 \\
\hline \multicolumn{10}{|c|}{ Grãos } \\
\hline \multicolumn{2}{|c|}{$45^{\circ} \mathrm{C}$} & \multicolumn{2}{|c|}{$60^{\circ} \mathrm{C}$} & \multicolumn{2}{|c|}{$75^{\circ} \mathrm{C}$} & \multicolumn{2}{|c|}{$90^{\circ} \mathrm{C}$} & \multicolumn{2}{|c|}{$105^{\circ} \mathrm{C}$} \\
\hline $\mathrm{X}^{*}$ & $\mathrm{r}$ & $\mathrm{X}^{*}$ & $\mathrm{r}$ & $\mathrm{X}^{*}$ & $\mathrm{r}$ & $\mathrm{X}^{*}$ & $\mathrm{r}$ & $\mathrm{X}^{*}$ & $\mathrm{r}$ \\
\hline 0,50 & 13,99 & 0,50 & 13,88 & 0,50 & 13,96 & 0,50 & 13,95 & 0,50 & 13,96 \\
\hline 0,41 & 13,83 & 0,40 & 13,67 & 0,40 & 13,71 & 0,40 & 13,68 & 0,41 & 13,70 \\
\hline 0,33 & 13,70 & 0,34 & 13,57 & 0,31 & 13,64 & 0,33 & 13,59 & 0,34 & 13,61 \\
\hline 0,27 & 13,65 & 0,26 & 13,49 & 0,27 & 13,56 & 0,27 & 13,54 & 0,27 & 13,52 \\
\hline 0,20 & 13,59 & 0,21 & 13,44 & 0,21 & 13,49 & 0,21 & 13,46 & 0,20 & 13,46 \\
\hline 0,15 & 13,55 & 0,16 & 13,38 & 0,15 & 13,44 & 0,15 & 13,39 & 0,14 & 13,40 \\
\hline 0,10 & 13,49 & 0,10 & 13,33 & 0,10 & 13,38 & 0,09 & 13,35 & 0,09 & 13,36 \\
\hline
\end{tabular}

Fonte: Elaboração dos autores.

$\mathrm{Na}$ Figura 4, são apresentados os valores do coeficiente de difusão efetivo para grãos de pinhãomanso, considerando o raio equivalente constante e variável, após à secagem em diferentes condições de ar.

Verifica-se na Figura 4 que, a difusividade efetiva dos grãos de pinhão-manso aumenta com o aumento da temperatura do ar de secagem, como já descrito por vários pesquisadores (VERA et al., 2005; CORRÊA et al., 2006a; RESENDE et al., 2007; GONELI et al., 2007; GELY; SANTALLA, 2007; RESENDE et al., 2009; ALMEIDA et al.,
2009; SOUSA et al., 2011; COSTA et al., 2011). Quanto maior a temperatura do ar de secagem, menor a resistência do grão a remoção de água, logo a difusividade é maior. Segundo Goneli et al. (2009), com o aumento da temperatura, a viscosidade da água diminui e, sendo a viscosidade uma medida de resistência do fluído ao escoamento, variações dessa propriedade implicam em alterações na difusão da água nos capilares do grão de modo a favorecer a movimentação desse fluído no produto. 
Figura 3. Coeficiente de difusão efetivo $\left(\mathrm{m}^{2} \cdot \mathrm{s}^{-1}\right)$ obtidos para a secagem dos grãos de pinhão-manso nas temperaturas de $45,60,75,90$ e $105^{\circ} \mathrm{C}$.

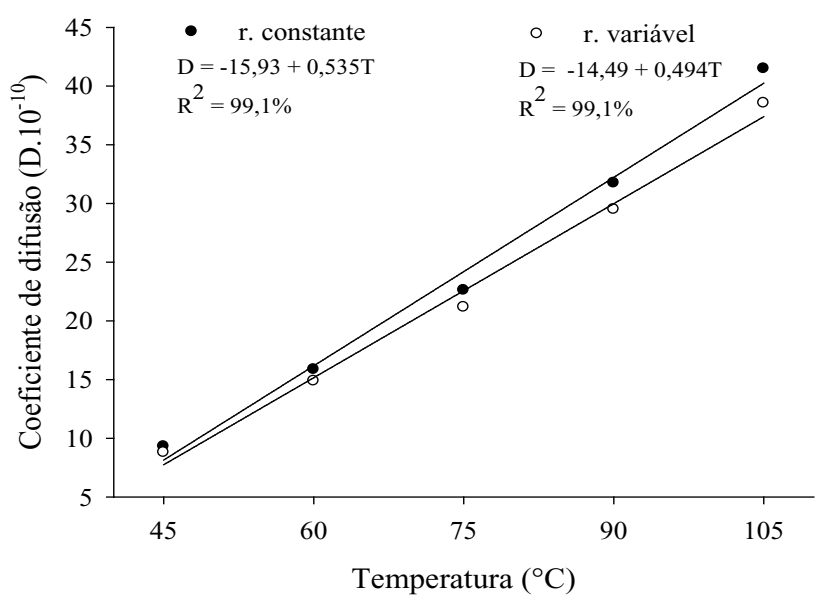

Fonte: Elaboração dos autores.

Os coeficientes de difusão dos grãos apresentaram magnitudes entre $9,29 \times 10^{-10}$ e $41,48 \times 10^{-10} \mathrm{~m}^{2} \cdot \mathrm{s}^{-1}$, utilizando-se o raio equivalente inicial dos grãos, ou seja, desprezando-se a redução de volume do produto ao longo da secagem, e entre $8,79 \times 10^{-10}$ e 38,54 x $10^{-10} \mathrm{~m}^{2} \cdot \mathrm{s}^{-1}$, considerando a contração volumétrica do grão. Logo, quando se emprega o fenômeno da contração há uma redução da difusividade efetiva dos grãos de pinhão-manso, independentemente da condição de secagem. O mesmo comportamento foi observado para a difusividade efetiva dos frutos de pinhão-manso (Figura 5), que apresentaram magnitudes entre $16,20 \times 10^{-10}$ e $68,11 \times 10^{-10} \mathrm{~m}^{2} \cdot \mathrm{s}^{-1}$, quando não considerado a contração volumétrica, $\mathrm{e}$ entre $9,61 \times 10^{-10}$ e $39,32 \times 10^{-10} \mathrm{~m}^{2} \cdot \mathrm{s}^{-1}$, considerandose a redução do volume do fruto.

Verifica-se nas Figuras 4 e 5 que, a difusividade efetiva é menor quando considerado o fenômeno da contração volumétrica, sendo esta diferença mais evidenciada na secagem dos frutos de pinhãomanso, em decorrência da maior redução do raio equivalente (Tabela 1). Corrêa et al. (2006a), trabalhando com grãos de feijão observaram magnitudes do coeficiente de difusão efetivo entre $2,21 \times 10^{-10}$ e $9,08 \times 10^{-10} \mathrm{~m}^{2} \cdot \mathrm{s}^{-1}$, considerando a contração volumétrica dos grãos e entre $2,77 \times 10^{-10}$ e $10,89 \times 10^{-10} \mathrm{~m}^{2} \cdot \mathrm{s}^{-1}$, utilizando o raio equivalente inicial dos grãos. Goneli et al. (2007), observaram magnitudes do coeficiente de difusão efetivo para grãos de trigo entre $8,3306 \times 10^{-11}$ e 41,0977 x 10${ }^{11} \mathrm{~m}^{2} \cdot \mathrm{s}^{-1}$ considerando-se a redução do volume do grão, e entre $9,0244 \times 10^{-11}$ e $44,6509 \times 10^{-11} \mathrm{~m}^{2} . \mathrm{s}^{-1}$ quando não considerado a contração volumétrica. Logo, a difusividade dos frutos e dos grãos de pinhão-manso é maior que a dos grãos de feijão e de trigo, comportamento esperado, pois a cultura em estudo é uma oleaginosa e o feijão e trigo são aleuro-amiláceo e amiláceo, respectivamente. Vale salientar que, produtos oleaginosos apresentam maior hidrofobicidade que aleuro-amiláceo e amiláceo, facilitando a remoção de água, aliado a isso, a faixa de temperatura do ar de secagem utilizada nos trabalhos com grãos de feijão e de trigo foi 25 a $55{ }^{\circ} \mathrm{C}$, enquanto que a do presente estudo é de 45 a $105^{\circ} \mathrm{C}$.

Os valores do coeficiente de difusão efetivo calculados para os grãos e frutos de pinhão-manso estão coerentes com os relatados na literatura para secagem de produtos agrícolas que, de acordo com Madamba, Driscoll e Buckle (1996), apresentam-se na ordem de $10^{-9}$ a $10^{-11} \mathrm{~m}^{2} . \mathrm{s}^{-1}$. 
Figura 4. Coeficiente de difusão efetivo $\left(\mathrm{m}^{2} \cdot \mathrm{s}^{-1}\right)$ obtidos para a secagem dos frutos de pinhão-manso nas temperaturas de $45,60,75,90$ e $105^{\circ} \mathrm{C}$.

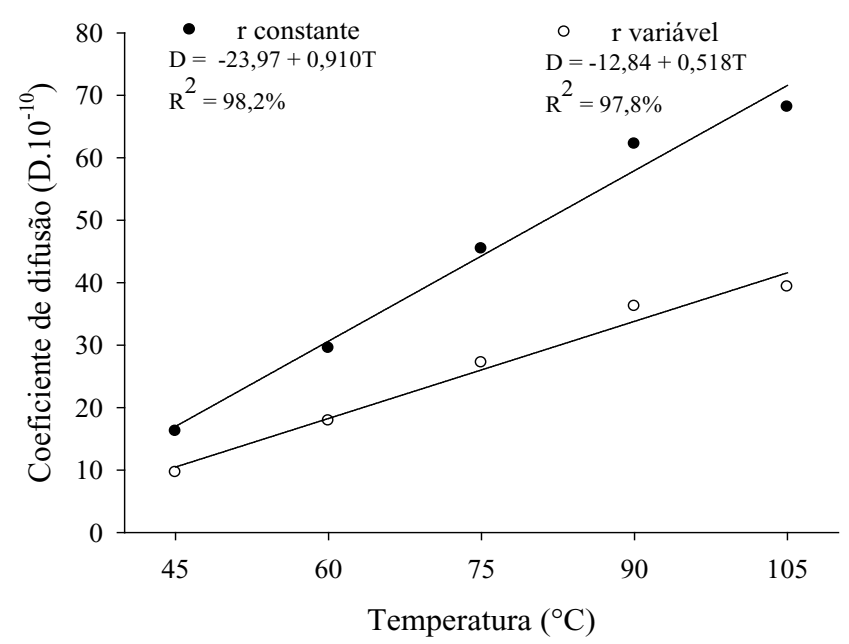

Fonte: Elaboração dos autores.

Analisando as Figuras 4 e 5, nota-se que a difusividade efetiva dos frutos de pinhão-manso é maior que a dos grãos. Este comportamento está relacionado principalmente com o teor de água desses materiais. Como o teor de água dos frutos era expressivamente maior que o dos grãos, existia uma maior quantidade de água livre, fazendo com que a velocidade de remoção de água em uma mesma área para um mesmo intervalo de tempo fosse maior para os frutos que para os grãos.

De acordo com o teste de identidade de modelo, os valores de $\chi_{\text {calculados }}^{2}$ foram superiores aos valores de $\chi_{\text {tabelados }}^{2}$, para o coeficiente de difusão efetivo dos grãos e dos frutos de pinhão-manso. Assim, rejeita-se a hipótese formulada $\mathrm{H}_{\mathrm{o}}$, ou seja, os modelos lineares utilizados para a representação desse fenômeno, diferem estatisticamente entre si, não sendo possível utilizar um único modelo para representar a variação do coeficiente de difusão efetivo em função da temperatura. Logo, é possível afirmar que a redução do volume altera significativamente a difusividade efetiva dos grãos e dos frutos de pinhão-manso. Sendo assim, torna-se necessário a inclusão da contração volumétrica para um melhor ajuste do modelo de difusão na secagem de grãos e de frutos de pinhão-manso.

A dependência do coeficiente de difusão efetivo dos grãos e dos frutos de pinhão-manso com relação à temperatura do ar de secagem foi satisfatoriamente representada pela expressão de Ahrrenius, conforme ilustrado na Figura 6. Sendo os parâmetros da energia necessária para desencadear o processo de secagem dos grãos e dos frutos de pinhão-manso, considerando-se ou não a contração volumétrica durante a secagem apresentados na Tabela 2.

Termodinamicamente, energia da ativação representa a energia necessária para o rompimento da barreira que as moléculas de água encontram, durante o processo de secagem, ao migrarem do interior para a superfície do produto, sendo que energia de ativação mais baixa traduz-se em maior difusividade de água no produto (SHARMA; PRASAD, 2004). Sendo assim, quanto menor a energia de ativação maior será a velocidade com que a água será removida do material, indicando que os produtos com maiores teores de água, apresentarão menor energia de ativação. 
Figura 5. Representação de Arrhenius para o coeficiente de difusão efetivo em função da temperatura do ar de secagem, obtido durante a secagem dos grãos e dos frutos de pinhão-manso.
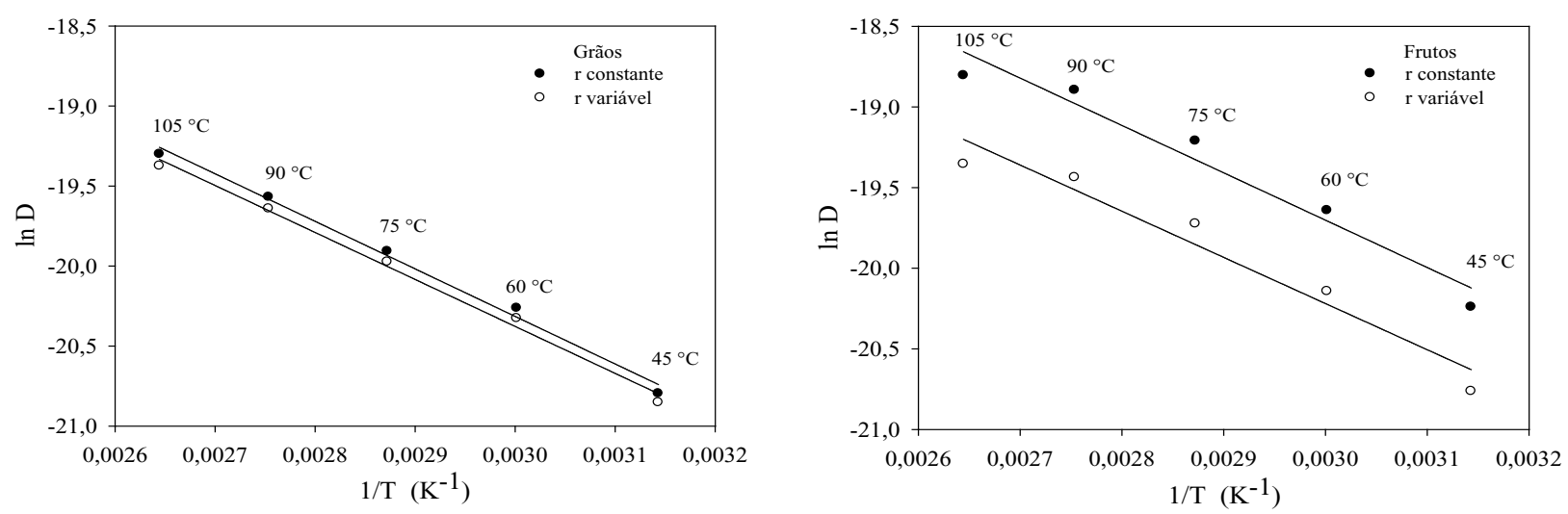

Fonte: Elaboração dos autores.

Verifica-se na Tabela 2, que os valores da energia de ativação dos grãos e dos frutos de pinhão-manso são muito próximos, sendo a dos grãos levemente superiores à dos frutos, pois apresentam menor teor de água. Nota-se ainda que, quando desconsiderado a redução do volume dos materiais a energia de ativação apresenta valores sensivelmente maiores, indicando que o fenômeno da contração volumétrica não exerce grande influência nesta avaliação.

Tabela 2. Energia de ativação $\left(\mathrm{kJ} \mathrm{mol}^{-1}\right)$, dos grãos e dos frutos de pinhão-manso, submetidos à secagem em cinco condições de ar.

\begin{tabular}{|c|c|c|c|}
\hline \multicolumn{2}{|c|}{ Grãos } & \multicolumn{2}{|c|}{ Frutos } \\
\hline r constante & r variável & r constante & r variável \\
\hline 24,17 & 23,87 & 23,88 & 23,29 \\
\hline
\end{tabular}

Fonte: Elaboração dos autores.

$\mathrm{Na}$ literatura pesquisada, foram encontrados valores da energia de ativação para diversos produtos agrícolas: para arroz cozido de 36,44 kJ.mol-1 (RAMESH, 2003), trigo 37,013 kJ.mol-1 (MOHAPATRA; RAO, 2005), feijão 40,08 kJ.mol-1 (CORRÊA et al., 2006b), trigo $42,00 \mathrm{~kJ} \mathrm{~mol}^{-1}$ (GONELI et al., 2007), feijão adzuki $31,16 \mathrm{~kJ} \mathrm{~mol}^{-1}$ (ALMEIDA, et al., 2009). Nota-se que a energia de ativação dos grãos e dos frutos de pinhãomanso estão inferiores aos produtos citados, este comportamento pode estar relacionado com a ligação mais instável da água com o componentes químicos dos materiais utilizados neste estudo.

\section{Conclusão}

Com base nos resultados obtidos conclui-se que:

- os coeficientes de difusão dos grãos apresentaram magnitudes entre 9,29 x $10^{-10}$ e 41,48 x $10^{-10} \mathrm{~m}^{2} \cdot \mathrm{s}^{-1}$ (sem contração) e, entre $8,79 \times 10^{-10} \mathrm{e}$ $38,54 \times 10^{-10} \mathrm{~m}^{2} . \mathrm{s}^{-1}$ (com contração volumétrica). Já os coeficientes de difusão dos frutos se apresentaram na ordem de $16,20 \times 10^{-10}$ e $68,11 \times 10^{-10} \mathrm{~m}^{2} \cdot \mathrm{s}^{-1}$ ( $\mathrm{sem}$ contração) e, $9,61 \times 10^{-10}$ e $39,32 \times 10^{-10} \mathrm{~m}^{2} \cdot \mathrm{s}^{-1}$ (com contração volumétrica).

- o coeficiente de difusão efetivo foi menor quando considerado o fenômeno da contração 
volumétrica, tanto para os grãos como para os frutos de pinhão-manso.

- o coeficiente de difusão efetivo dos grãos e dos frutos de pinhão-manso aumentou com a elevação da temperatura do ar;

- a inclusão da contração volumétrica promoveu um melhor ajuste do modelo da difusão líquida;

- a energia de ativação para a difusão líquida dos grãos de pinhão-manso foi de $24,17 \mathrm{~kJ} \cdot \mathrm{mol}^{-1} \mathrm{e}$ a dos frutos de 23,88 kJ.mol ${ }^{-1}$, quando desconsiderado a contração volumétrica. Quando acrescentou-se o fenômeno da contração a energia de ativação foi de 23,87 e $23,29 \mathrm{~kJ} \cdot \mathrm{mol}^{-1}$, para grãos e frutos, respectivamente.

- a energia de ativação dos grãos e dos frutos de pinhão-manso é similar e a inclusão da contração volumétrica praticamente não altera este parâmetro.

\section{Agradecimentos}

Ao $\mathrm{CNPq}$ e CAPES pelo apoio financeiro indispensável a execução do presente trabalho.

\section{Referências}

ALMEIDA, D. P.; RESENDE, O.; COSTA, L. M.; MENDES, U. C.; SALES, J. F. Cinética de secagem do feijão adzuki (Vigna angularis). Global Science and Technology, Rio verde, v. 2, n. 1, p. 72-83, 2009.

BRASIL, Ministério da Agricultura Pecuária e Abastecimento. Secretaria de defesa Agropecuária. Regras para análise de sementes. Brasília: Mapa/ACS, 2009. $395 \mathrm{p}$.

CORRÊA, P. C.; RESENDE, O.; GONELI, A. L. D.; BOTELHO, F. M.; NOGUEIRA, B. L. Determinação do coeficiente de difusão líquida dos grãos de feijão. Revista Brasileira de Produtos Agroindustriais, Campina Grande, v. 8, n. 2, p. 117-126, 2006 a.

CORRÊA, P. C.; RIBEIRO, D. M.; RESENDE, O.; BOTELHO, F. M. Determinação e modelagem das propriedades físicas e da contração volumétrica do trigo durante a secagem. Revista Brasileira de Engenharia Agrícola e Ambiental, Campina Grande, v. 10, n. 3, p. 665-670, 2006b.
COSTA, L. M.; RESENDE, O.; SOUSA, K. A.; GONÇALVES, D. N. Coeficiente de difusão efetivo e modelagem matemática da secagem de sementes de crambe. Revista Brasileira de Engenharia Agrícola e Ambiental, Campina Grande, v. 15, n. 10, p. 1089-1096, 2011.

GELY, M. C.; SANTALLA, E. M. Moisture diffusivity in quinoa (Chenopodium quinoa Willd.) seeds: Effect of air temperature and initial moisture content of seeds. Journal of Food Engineering, Essex, v. 78, n. 3, p. 10291033, 2007.

GONELI, A. L. D.; CORRÊA, P. C.; AFONSO JÚNIOR, P. C.; OLIVEIRA, G. H. H. Cinética de secagem dos grãos de café descascados em camada delgada. Revista Brasileira de Armazenamento, n. 11, p. 64-73, 2009. Especial Café.

GONELI, A. L. D.; CORREAA, P. C.; RESENDE, O.; REIS NETO, S. A. Estudo da difusão de umidade em grãos de trigo durante a secagem. Ciência Tecnologia de Alimentos, Campinas, v. 27, n. 1, p. 135-140, 2007.

KOÇ, B.; EREN, I.; ERTEKIN F. K. Modelling bulk density, porosity and shrinkage of quince during drying: The effect of drying method. Journal of Food Engineering, Essex, v. 85, n. 3, p. 340-349, 2008.

LAHSASNI, S.; KOUHILA, M.; MAHROUZ, M.; JAOUHARI, J. T. Drying kinetcs of prickly pear fruit (Opuntia ficus indica). Journal of Food Engineering, Essex, v. 2, n. 61, p. 173-179, 2004.

MADAMBA, P. S.; DRISCOLL, R. H.; BUCKLE, K. A. Thin-layer drying characteristics of garlic slices. Journal of Food Engineering, Essex, v. 29, n. 1, p. 75-97, 1996.

MIRANDA, M.; MAUREIRA, H.; RODRIGUEZ, K.; VEGA-GALVEZ, A. Influence of temperature on the drying kinetics, physicochemical properties, and antioxidant capacity of Aloe Vera (Aloe barbadensis Miller) gel. Journal of Food Engineering, Essex, v. 91, n. 2, p. 297-304, 2009.

MOHAPATRA, D.; RAO, P. S. A thin layer drying model of parboiled wheat. Journal of Food Engineering, Essex, v. 66, n. 4, p. 513-518, 2005.

MOHSENIN, N. N. Physical properties of plant and animal materials. New York: Gordon and Breach Publishers, 1986. $841 \mathrm{p}$.

OLIVEIRA, R. A.; OLIVEIRA, W. P.; PARK, K. J. Determinação da difusividade efetiva de raiz de chicória. Engenharia Agrícola, Jaboticabal, v. 26, n. 1, p. 181-189, 2006. 
RAMESH, M. N. Moisture transfer properties of cooked rice during drying. Journal of Food Science, Chicago, v. 36, n. 2, p. 245-255, 2003.

RAMOS, I. N.; BRANDÃO, T. R. S.; SILVA, C. L. M. Integrated approach on solar drying, pilot convective drying and microstructural changes. Journal of Food Engineering, Essex, v. 67, n. 1-2, p. 195-203, 2005.

REGAZZI, A. J. Teste para verificar a igualdade de parâmetros e a identidade de modelos de regressão não linear. Revista Ceres, Viçosa, v. 50, n. 287, p. 9-26, 2003.

RESENDE, O.; CORRÊA, P. C.; JARÉN, C.; MOURE, A. J. Bean moisture diffusivity and drying kinetics: a comparison of the liquid diffusion model when taking into account and neglecting grain shrinkage. Spanish Journal of Agricultural Research, Madrid, v. 5, n. 1, p. 51-58, 2007.

RESENDE, O.; RODRIGUES, E.; MENDES, U. C.; ALMEIDA, D. P. Hidratação e difusividade efetiva do feijão adzuki (Vigna angularis). Revista Brasileira de Armazenamento, Viçosa, v. 34, n. 2, p. 122-128, 2009.

SHARMA, G. P.; PRASAD, S. Effetive moisture diffusivity of garlic cloves undergoing microwaveconvective drying. Journal of Food Engineering, Essex, v. 65 , n. 4 , p. 609-617, 2004.
SIRISOMBOON, P.; KITCHAIYA, P. Physical properties of Jatropha curcas L. kernels after heat treatments. Biosystems Engineering, London, v. 102, n. 2, p. 244250, 2009.

SOUSA, K. A.; RESENDE, O.; CHAVES, T. H.; COSTA, L. M. Cinética de secagem do nabo forrageiro (Raphanus sativus L.). Revista Ciência Agronômica, Fortaleza, v. 42, n. 4, p. 883-892, 2011.

ULLMANN, R.; RESENDE, O.; SALES, J. F.; CHAVES, T. H. Qualidade das sementes de pinhão manso submetidas à secagem artificial. Revista Ciência Agronômica, Fortaleza, v. 41, n. 3, p. 442-447, 2010.

VEGA-GÁLVEZ, A.; MIRANDA, M.; CLAVERÍA, R.; QUISPE, I.; VERGARA, J.; URIBE, E.; PAEZ, H.; SCALA, K. D. Effect of air temperature on drying kinetics and quality characteristics of osmo-treated jumbo squid (Dosidicus gigas). Food Science and Technology, Amsterdam, v. 44, n. 1, p. 16-23, 2011.

VERA, C. M.; MENDOZA, M. G. V.; ESPINOSA, H.; CABALLERO, F. Moisture diffusion coefficient of amaranth seeds determined by the regular regime method. Biosystems Engineering, London, v. 92, n. 4, p. 439-443, 2005. 
